\title{
Novel anti-cancer compounds: Structure-based discovery of chemical chaperons for p53
}

\author{
YUMIKO OKUDA, HIRONORI K. NAKAMURA and KAZUO KUWATA \\ Division of Prion Research, Center for Emerging Infectious Diseases, \\ Graduate School of Medicine, Gifu University, 1-1 Yanagido, Gifu 501-1194, Japan
}

Received March 10, 2009; Accepted May 8, 2009

DOI: 10.3892/or_00000495

\begin{abstract}
Thermal stability of p53 is crucial in preventing cancer proliferation. Critical mutations which significantly destabilize p53 conformation prevent normal interaction between p53 and DNA and consequently interfere with its inhibitory function against cancer proliferation. The purpose of this study was to discover the small compounds called 'chemical chaperons' that can efficiently stabilize the functional p53 conformation and restore the anti-cancer activity. To search for such compounds, we performed a docking simulation using the AutoDock program and the ZINC database. Simply based on the docking energy, we extracted 70 compounds (GJC1-GJC70) and examined their anti-cancer activity using the MTT assay of the human colon cancer cells, HCT116. We found that two compounds, GJC29 and GJC30, significantly inhibited the proliferation of cancer cells compared to the positive control staurosporine. Interaction between p53 and novel anti-cancer compounds were confirmed using SPR measurements. Intriguingly, in the simulated binding mode, both compounds bind to the pocket in the vicinity of the residue V143, one of the mutation hot-spots in p53. Finally, we injected each compound subcutaneously into the nude mice implanted with HCT116 and found that GJC29 has a strong suppressive effect against cancer proliferation in vivo. In conclusion, p53 is an appropriate target for the rational design of the chemical chaperon for cancer treatment.
\end{abstract}

\section{Introduction}

p53 is a tumor suppressor, i.e. a potent inducer of apoptosis and cell cycle arrest. In particular, DNA damage and aberrantly activated oncogenes activate p53 to prevent the emergence of cancer cells (1). The importance of p53 in preventing cancer is supported by the fact that p53 is the most frequently mutated gene observed in human cancers. Approximately $50 \%$ of all

Correspondence to: Dr Kazuo Kuwata, Division of Prion Research, Center for Emerging Infectious Diseases, Graduate School of Medicine, Gifu University, 1-1 Yanagido, Gifu 501-1194, Japan E-mail: kuwata@gifu-u.ac.jp

Key words: colon cancer, anti-cancer drug, p53, structure-based drug discovery human sporadically arising cancers incur loss or mutation in the p53 gene and it has been estimated that at least $80 \%$ of all human cancers have dysfunctions of p53 $(2,3)$. Thus, restoration of normal p53 function may be an important strategy for the cancer treatment.

p53 consists of three domains: the N-terminal domain which activates the transcription, the DNA binding domain and the C-terminal domain which is responsible for the tetramer formation, as illustrated in Fig. 1a. Intriguingly, causative mutations of p53 protein are concentrated on the DNA binding domain which is well conserved over various species and thus it is called a 'hot-spot' (4). On the other hand, p53 is known to be one of the natively unfolded proteins (5-8) and it folds only upon binding with DNA. However, if p53 has a mutation at one of the critical regions, it may not fold properly and lose the normal function of inducing apoptosis, cell cycle arrest and allow the progression of cancer (8).

In general, normal p53 function is impaired in almost all the cancer cells, implying that the population of normallystructured p53 decreases because of various reasons, c.f. low expression of $\mathrm{p} 53$, any mutation at one of the critical regions, environmental alterations to hamper the $\mathrm{p} 53$ folding process resulting in the activation of ubiquitine-dependent degradation pathway (9) and so on.

Hopefully, we may be able to increase the population of functional p53 by stabilizing its folded conformation using a designed compound termed 'chemical chaperon' (10-23), thereby inducing the apoptosis and cell cycle arrest. Here we tried to design such compounds rationally and to suppress the proliferation of colon cancer HCT116 cells.

Initially we employed the in silico screening to discover chemical chaperons based on the three dimensional structure of p53 forming a complex with DNA (24). The chemical chaperons are expected to stabilize the functional conformation of p53, as in case of other conformational diseases, such as prion diseases (10) or cancer (11-23) and restore its native conformation. Then, we examined their anti-cancer effects using ex vivo screening, the binding assay using surface plasmon resonance (SPR) and in vivo screening, according to the general flow of rational drug design illustrated in Fig. 1b.

\section{Materials and methods}

In silico screening. The lead-like subset ( 600,000 compounds) of the ZINC database (25) was used for docking simulations. 
a

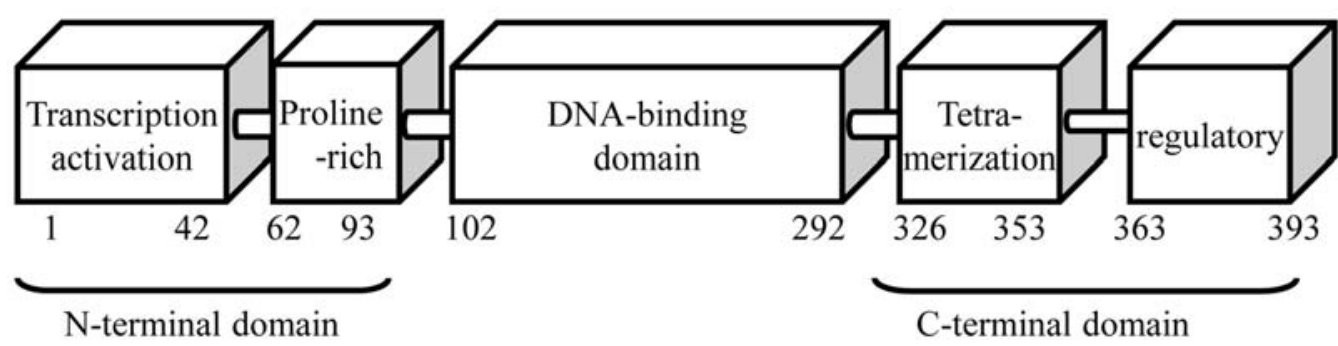

b

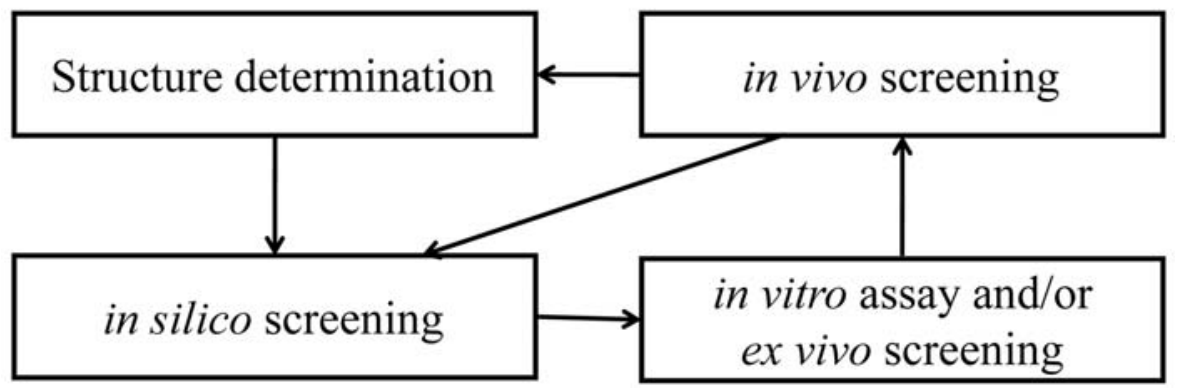

Figure 1. (a) Functional domains in p53 molecule. N-terminal domain of the p53 includes the region for transcriptional activity, while C-terminal domain includes a region for tetramer formation. (b) General scheme of the structure-based drug discovery (SBDD) utilized for the design of a chemical chaperon. Here we started from the in silico screening because the target structure was already determined (24).

The flexibility of compounds were determined automatically using AUTOTORS, which is a program of the AutoDock package (26) and the united-atom model for the compound was created also with AUTOTORs: Non-polar hydrogen atoms of compounds were deleted after the partial charge of nonpolar hydrogen atom was moved to a carbon atom bonded covalently, where the original charge information was given in the ZINC database. Chain A picked up from 1TUP (PDB code) was used for the target structure of p53. AutoDockTools were used for giving charge information (Kollman unitedatom charge) and building an united-atom model for the protein. The solvation parameters were set automatically using ADDSOL, which is also a program of the AutoDock package. The dimensions of the grids for the docking were with $120 \times 120 \times 120$ points $(45 \AA * 45 \AA * 45 \AA)$, a grid-point spacing of $0.375 \AA$. The center position of grid box was set to the center of mass of the protein. AutoDock ver. 3.05 was used for automated docking simulation (25). Standard parameters were used, which were created with mkdpf3, a script program of AutoDock package: a population size of 50, a random starting position and conformation, a maximal mutation of $2 \AA$ in translation and 50 degrees in rotations, an elitism of 1 , a mutation rate of 0.02 , a crossover rate of 0.8 , runs of 10 times, energy evaluations of 250,000 , selecting the Lamarckian genetic search algorithm (LGA). Calculations were performed with a 26-node PC-cluster where each node has a dual core CPU (Intel Pentium-D 2.8GHz). Therefore, 52 parallel calculations can be performed without large loss of calculation efficiency in the PC-cluster.

p53. Recombinant full length p53 (residue 1-393) was purchased from Santa Cruz Biotechnology (Lot \# L11305). This construct includes all the elements shown in Fig. 1 b.
Compounds. We selected compounds depending on the lowest docked energy among 10 runs. Seventy compounds were purchased from ASINEX (Russia) and Enamine (Ukraine) and tested for anti-cancer activity.

Ex vivo experiment. HCT116, colon cancer cells with wildtype p53 were purchased from ATCC (American type culture collection, Manassus, VA). Cells used in this study were maintained in either DMEM or McCoy's 5A medium, both supplemented with $10 \% \mathrm{FBS}$, penicillin $(120 \mu \mathrm{g} / \mathrm{ml})$ and streptomycin $(200 \mu \mathrm{g} / \mathrm{ml})$ at $37^{\circ} \mathrm{C}$ in a $5 \% \mathrm{CO}_{2}$ atmosphere. Cells were seeded into 96 -well plates with an initial density of $0.5 \times 10^{4}$ cells per well of $100 \mu 1$ volume and grown for two days. Compounds were dissolved in DMSO at a concentration of $1 \mathrm{mM}$ and administered $1 \mu 1$ of DMSO solution to $99 \mu 1$ of HCT116 cells. Thus the final concentration of compounds was $10 \mu \mathrm{M}$. After incubation for an additional $24 \mathrm{~h}$, cell viability was determined with the MTT assay (Roche, Cell Proliferation Kit). In the MTT assay, absorbance at $550 \mathrm{~nm}$ was detected to determine the formazan level.

Interaction of compound with p53. To determine the binding affinity of compounds with p53, we used the surface plasmon resonance (SPR). All SPR measurements were performed using the BIAcore-T100 apparatus (GE Healthcare, Sweden) at $25^{\circ} \mathrm{C}$. We used the sensor chip CM5 for all the experiments. The immobilization of p53 was performed using the amine coupling kit at a flow rate $10 \mu \mathrm{l} / \mathrm{min}$. We used HBS-EP+ (0.01 M HEPES pH 7.4, 0.15 M NaCl, 3 mM EDTA) (pH 7.4) in the presence of $0.005 \%$ surfactant, P20 (polyoxyethylene sorbitan monolaurate) for running buffer. The dextran on the chip was initially equilibrated with running buffer and carboxymethylated matrix was activated with an EDC/NHS 
(a)

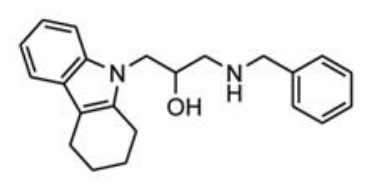

(b)<smiles>Cc1cc(=O)oc2cc(OCC(=O)Nc3ccc4c(c3)CCC4)ccc12</smiles>

(c)

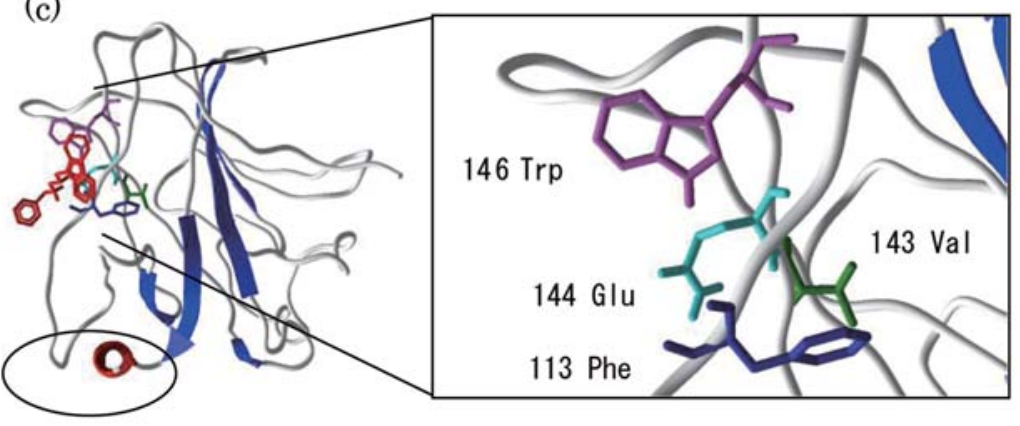

DNA binding region

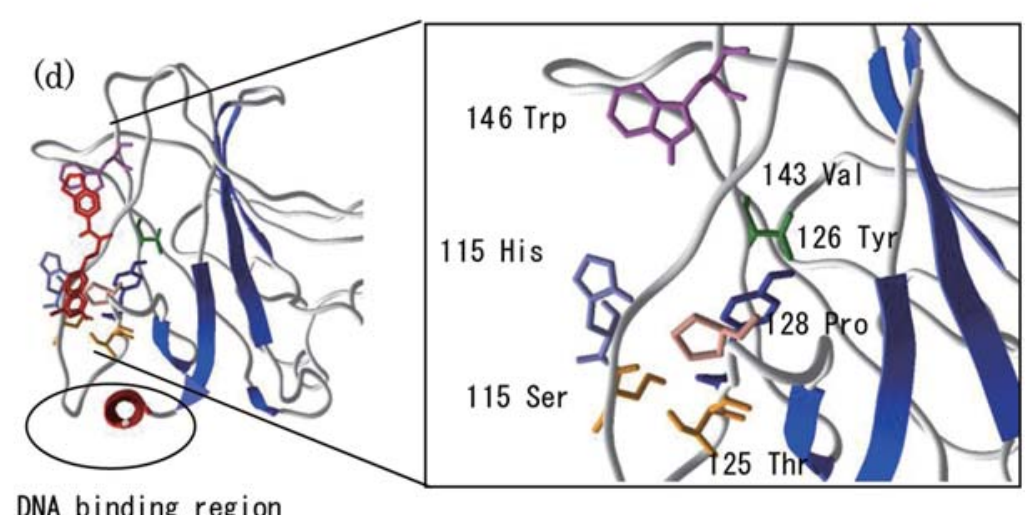

Figure 2. Chemical structures of (a) GJC29 and (b) GJC30 and binding modes (c) between GJC29 and p53, and (d) between GJC30 and p53, which were obtained by the docking simulation using AutoDock.

mixture. p53 $(100 \mu \mathrm{l})(50 \mu \mathrm{g} / \mathrm{ml})$ in $5 \mathrm{mM}$ sodium acetate ( $\mathrm{pH} 4.0)$ was injected for coupling reaction and finally unreacted carboxymethylated groups were blocked by injecting ethanolamine ( $\mathrm{pH} 8.5)$. The responses for immobilized $\mathrm{p} 53$ were around 2000 Response Unit (RU). The dissociation constants of small compounds were obtained by fitting RU values at different compound concentrations to the theoretical curve assuming a single binding site using the BIA evaluation 3.0 software.

In vivo experiment. Human colon cancer HCT116 cells $\left(2.5 \times 10^{7} / 200 \mu \mathrm{l}\right)$ were implanted to the left and right lateral abdominal regions of nude mice by a subcutaneous injection, as shown in Fig. 5a. At three weeks after the implantation, the average size of tumors reached $0.5 \mathrm{~cm}^{3}$. For in vivo treatment, compounds were suspended in saline with a concentration of $1 \mathrm{mM}$. Then $5 \mathrm{mg} / \mathrm{kg}$ of the solution was administered to each cancerous region every day by subcutaneous injection. Then tumor volumes were monitored also each day.

\section{Results}

In silico screening. We selected 70 compounds based on the docked energy among 10 runs. Docked energy of these compounds were almost the same: The lowest and highest ones were -11.85 to -10.65 , respectively. However, their binding modes are quite different. In Fig. 2, the structure of two hit compounds in ex vivo screening and their binding modes are shown. Intriguingly, both compounds bind with residues around the pocket which is located near a hot-spot, V143 $(4,14)$. In GJC29 side chain of W146 is expected to be stacked on the carbazole rings of GJC29, possibly by a $\pi-\pi$ interaction and E144 side chain also interacts with the carbazole moiety, possibly by $\mathrm{CH}-\pi$ interactions, aromatic ring of the other side of GJC29 is exposed to the solvent. In GJC30, W146 also stacks with the similar indole ring of GJC30 and the other aromatic rings interact with the H115 side chains both by the $\pi-\pi$ interactions.

Ex vivo screening. Then we conducted ex vivo screening using HCT116 cells. In MTT assay, the absorbance at $550 \mathrm{~nm}$ in case of positive control, i.e. staurosporin was $0.092 \pm 0.007$ as shown in Fig. 3. Among 70 selected compounds, GJC29 and GJC30 significantly suppressed the proliferation of cancer cells compared to staurosporine as indicated by the absorbance intensity shown in Fig. 3.

Interaction with $p 53$. The interactions between compounds and p53 were monitored with SPR. Fig. $4 \mathrm{a}$ and b shows the binding isotherm for GJC29 and GJC30, respectively, using RU values. Although the contribution of non-specific interaction was hard to remove in the case of GJC29, apparent dissociation constants for GJC29 and GJC30 were 


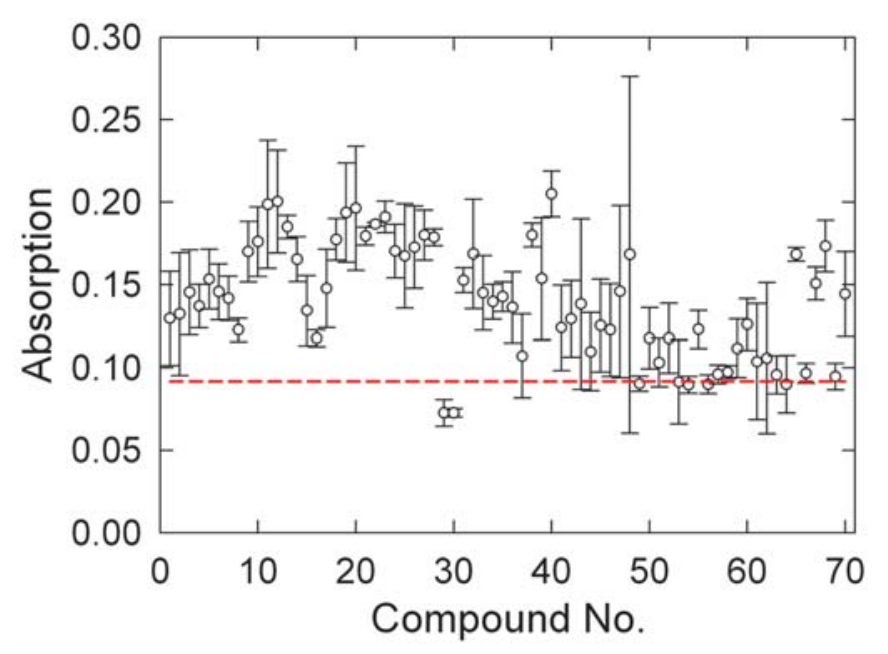

Figure 3. Ex vivo experiment using MTT assay. Absorbance intensity at $550 \mathrm{~nm}$ in the MTT assay for positive control, staurosporine was $0.092 \pm 0.007$ (shown by the red line). Among 70 selected compounds (GJC1 to GJC70), GJC29 and GJC30 significantly inhibited the proliferation of cancer cells compared to staurosporine.

roughly estimated to be 2.6 and $2.0 \mathrm{mM}$, respectively. In case of GJC29, binding site may not be unique but may promote the p53 folding upon binding (cooperative binding).

In vivo screening. We examined whether GJC29 and GJC30 could suppress the growth of tumor xenografts in nude mice implanted with human colon cancer, HCT116. Three weeks after the implantation of HCT116 cells, tumors attained a mass of $\sim 500 \mathrm{mg}$. Then they were treated by subcutaneous injection of saline, GJC29, GJC30 or staurosporine dissolved in $0.9 \%$ saline. Four mice were treated with saline (control) and no mice died though the experiment. Five mice were treated with staurosporine and one mouse died the 11th day. Four mice were treated with GJC29 and two mice died at the 5 th and 12th day, respectively. Five mice were treated with GJC30, and two mice died at the 11th day. In Fig. 5b and c, average tumor volumes are plotted as a function of days after subcutaneous injection. In the group treated with saline or GJC29, the tumor size was gradually increased after injection (Fig. 5b), while in that with staurosporine, growth of tumor was moderately suppressed (Fig. 5c). On the other hand, in the group treated with GJC29, averaged tumor size was reduced dramatically (Fig. 5c).

\section{Discussion}

We discovered an anti-cancer substance, 1,2,3,4- tetrahydro- $\alpha$ [[(phenylmethyl)amino]methyl]-9H-carbozole 9-ethanol, termed GJC29 by performing a series of experiments according to the rational drug design scheme shown in Fig. 1b. Since three dimensional structure of p53 was already clarified (24), we started from the in silico screening of 600,000 compounds in the ZINC database and identified two compounds, GJC29 and GJC30, which suppressed the tumor growth in ex vivo experiments. We confirmed the direct binding of GJC29 with p53 using SPR. Then we finally confirmed that the small molecule GJC29 with molecular weight of 334, can suppress tumor growth in vitro. Meanwhile, in vivo experiments a

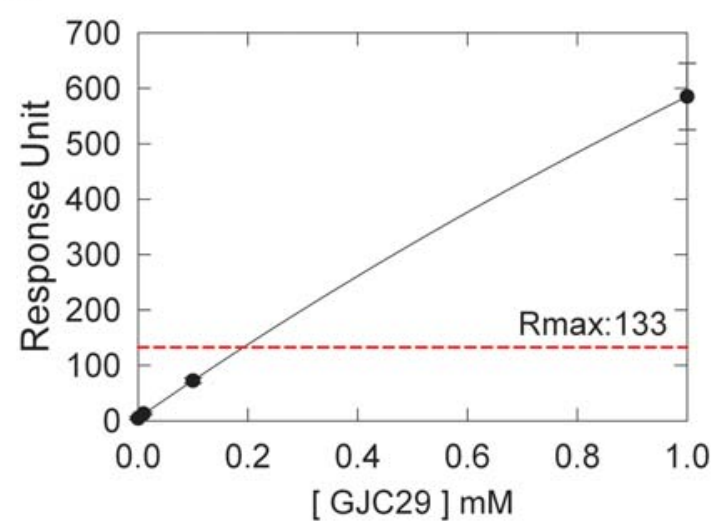

b

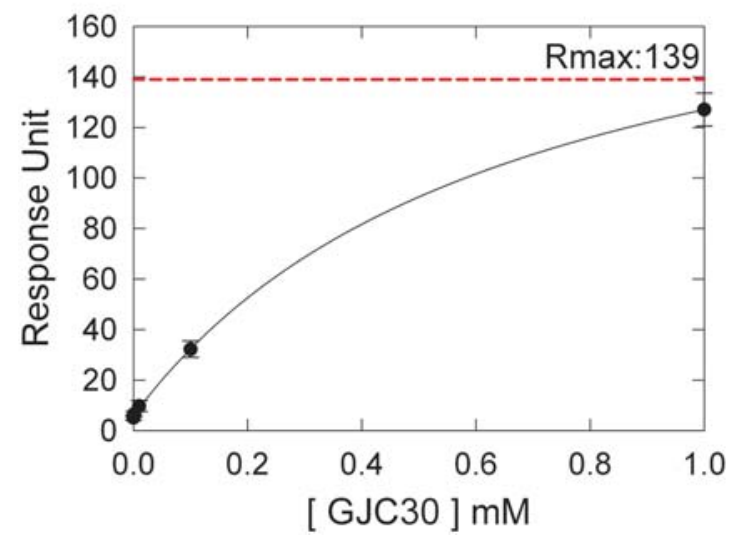

Figure 4. (a) Binding isotherm for the interaction between GJC29 and p53, obtained by SPR. RU values were obtained as a function of compound concentrations. $K_{\mathrm{d}}$ was estimated to be $2.6 \mathrm{mM}$ by the non-linear curve fit. Non-specific interaction is rather overwhelming (see text). (b) Binding isotherm for the interaction between GJC30 and p53, obtained by SPR. RU values were obtained as a function of compound concentrations. $K_{\mathrm{d}}$ was estimated to be $2.0 \mathrm{mM}$ by the non-linear curve fit.

showed that GJC29 administered only peripherally could profoundly reduce the size of the implanted human colon cancer cells in nude mice.

Development of the in silico screening techniques has enabled us to search for candidates for novel therapeutic drugs among over ten millions of compounds. Currently, 100,000 compounds per day could be tested via high throughput screening (HTS) technology, but it is almost impossible to test all compounds in a compound database, such as ACD (Available Chemicals Directory, MDL Information Systems), which includes over $10^{7}$ compounds.

To date, various in silico screening computer programs have been widely applied for the discovery of small compounds such as enzyme inhibitors, where the well-defined active sites usually serve as the target regions. Since p53 has not any catalytic activity, we did not assume any potential binding target region for in silico screening. Each compound in the database was evaluated through an analysis of the binding energy. Obtained compound GJC29 may stabilize the p53 conformation possibly through the specific binding with the region near the hot-spot, V143, as shown in Fig. 2a and works as a chemical chaperone. 
a

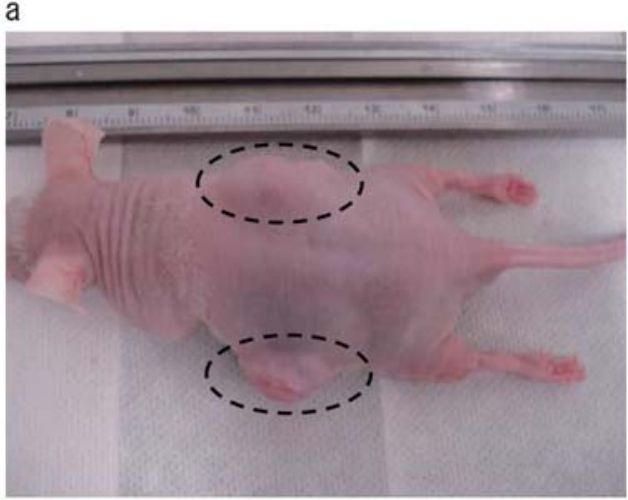

b

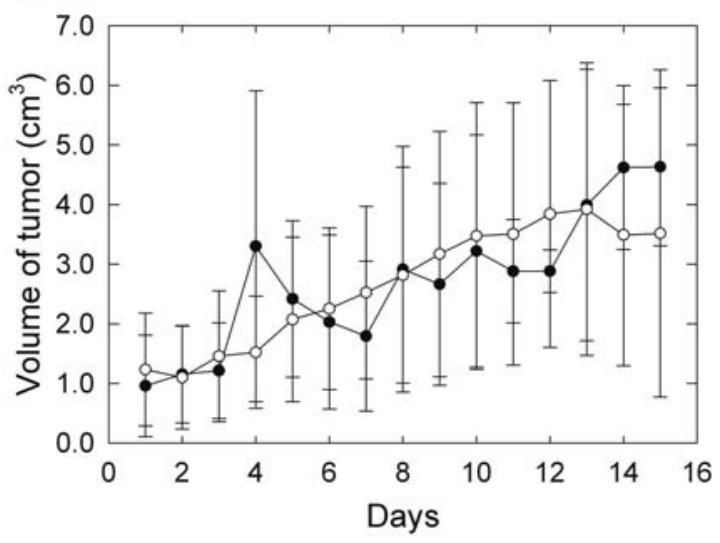

C

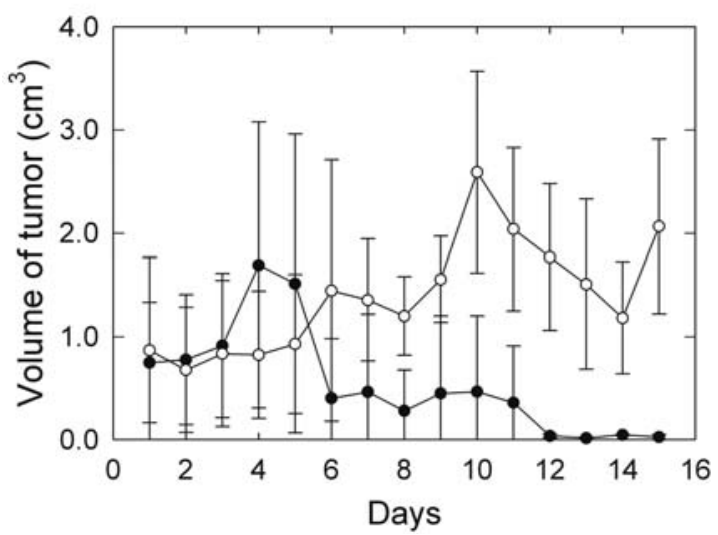

Figure 5. (a) A nude mouse at 3 weeks after implantation of cancer cells, HCT116. Cancer cells were implanted at right and left lateral abdominal regions as indicated by dotted circles. (b) In vivo experiment. We examined the effects of GJC29 or GJC30 on the growth of tumor xenograft in nude mice. The tumor volumes are plotted as a function of time after subcutaneous injection. In the control group treated with saline (o), GJC30 (•), the tumor size increased similarly with the date after injection. (c) Effects of staurosporine ( $)$ and GJC29 (•). In the group treated by GJC29, the tumor volume reduced significantly after the injection, even compared to the positive control, staurosporine.

The hit rate of the in silico screening in this study is $\sim 1.4 \%$. Thus it is conceivable that the in silico screening from huge compound libraries would be valuable for the discovery of the potential anti-cancer drugs.

Moreover, applying in silico screening in combination with ex vivo screening would provide us with a hint regarding the functional mechanism of p53, as well as the way of its regulation using small compounds. The calculated docked energy differences among selected compounds were quite small and not statistically significant. This is because the evaluation function utilized in the software was not necessarily designed for the interaction between p53 and small compounds. In view of the p53 folding reaction upon binding with DNA, it may be necessary to further evaluate the change in the entropy of solvation associated with binding and the change in the chain entropy of the p53. Rigorous evaluation of these effects in future would also help provide information on the physiological function of p53.

Calculated binding modes showed that both GJC29 and GJC30 bind with the pocket constructed by residues around V143, which correspond to one of the mutation hot-spots $(4,14)$. V143 is located in strand S3 of the B-sandwich and deeply buried to participate in the packing of the hydrophobic core (26). Truncation of two methyl groups from Val to Ala143 dramatically destabilized the structure by $3.34 \mathrm{kcal} / \mathrm{mol}$ (14). According to Bullock and Fersht (15), $20 \%$ of the V143A mutant is denatured at $37^{\circ} \mathrm{C}$. Since this mutation also causes the chemical-shift changes in almost all residues in the $\beta$-sandwich and the DNA-binding surface, long-range effects of V143A mutation may also affect the specificity of DNA binding. Increase of the thermal stability of this region is thus considered to be critical for the chemical chaperon activity. Effective compounds, GJC29 and GJC30 confirmed by ex vivo experiments may bind with the cavity formed by V143 and stabilize the p53 conformation.

More importantly, the docking simulation suggests that GJC29 and GJC30 intercalate amino and residue, W146 and E144, and W146 and H115 by $\pi-\pi$ interaction or $\mathrm{CH}-\pi$ interactions. These interactions would help stabilize the p53 conformation by reducing the global conformational fluctuation around biding sites.

Measurements of the dissociation constant $K_{\mathrm{d}}$ were complicated, as shown in Fig. 4a and b. In the case of GJC29, non-specificity is rather overwhelming and it was technically impossible to remove it. Sometimes this kind of nonspecificity must be avoided because many side effects may result from the non-specific binding with many physiologically important proteins other than $\mathrm{p} 53$, which is unfavorable from a pharmacological point of view. It may be conceivable that GJC29 induce the p53 folding thereby increasing the binding sites non-specifically. The cooperative binding may help stabilize the functional p53 conformation. We need to further optimize the chemical structure of the anti-cancer lead compound GJC29.

The discrepancy in the ex vivo and in vivo effects may be partly explained by the differences in the drug delivery efficiency and/or the metabolism between GJC29 and GJC30 in the body. For instance, xlog P-values for GJC29 and GJC30 are predicted to be 4.2 and 3.5 , respectively. Therefore, the former would be relatively easy to deliver to the intranuclear compartment where the compound is expected to interact with p53 molecules. This point is quite important and must be clarified further. Five mice died during the treatment with staurosporine, GCJ29 or GJC30 suggesting the toxicity of the three compounds. Thus side effects of the lead compound, GJC29 must be carefully examined before future optimization. 
Quite recently Boeckler et al (27) reported the anti-cancer compound, phikan 059, whose structure is somewhat similar to GJC29. The interaction sites between p53 and phikan 059 include W149 as evidenced by X-ray crystallography (24). However, unfortunately biological effects of phikan 059 were not examined (27). Thus, our study could be complementary to their study.

In conclusion, a novel anti-cancer compound was identified in our screening of the 600,000 compounds in the ZINC database. This potent inhibitor has chemical structures that may be easily optimized and those derivatives might become candidates of cancer therapeutics.

\section{Acknowledgements}

This study was supported by a research grant from the Aichi Cancer Center Hospital and Research Institute. We thank Dr Kiminori Kimura for technical assistance and also Dr Hideki Nikami for assistance in the in vivo experiments.

\section{References}

1. Vousden KH and Lu X: Live or let die: the cell's response to p53. Nat Rev Cancer 2: 594-604, 2002.

2. Levine AJ: p53, the cellular gatekeeper for growth and division. Cell 88: 323-331, 1997.

3. Lozano $\mathrm{G}$ and Elledge SJ: p53 sends nucleotides to repair DNA. Nature 404: 24-25, 2000.

4. Wong KB, DeDecker BS, Freund SM, Proctor MR, Bycroft M and Fersht AR: Hot-spot mutants of p53 core domain evince characteristic local structural changes. Proc Natl Acad Sci USA 96: 8438-8442, 1999.

5. Dyson HJ and Wright PE: Intrinsically unstructured proteins and their functions. Nat Rev Mol Cell Biol 6: 197-208, 2005.

6. Bullock AN, Henckel J, DeDecker BS, Johnson CM, Nikolova PV, Proctor MR, Lane DP and Fersht AR: Thermodynamic stability of wild-type and mutant p53 core domain. Proc Natl Acad Sci USA 94: 14338-14342, 1997.

7. Bell S, Klein C, Müller L, Hansen S and Buchner J: p53 contains large unstructured regions in its native state. J Mol Biol 322: 917-927, 2002.

8. Dawson R, Müller L, Dehner A, Klein C, Kessler H and Buchner J: The N-terminal domain of p53 is natively unfolded. J Mol Biol 332: 1131-1141, 2003.

9. Dornan D, Wertz I, Shimizu H, Arnott D, Frantz GD, Dowd P, Rourke KO, Koeppen H and Dixit VM: The ubiquitin ligase COP1 is a critical negative regulator of p53. Nature 429: 86-92, 2004.

10. Kuwata K, Nishida N, Matsumoto T, Kamatari Y, HosokawaMuto J, Kodama K, Nakamura HK, Kimura K, Kawasaki M, Takakura Y, Shirabe S, Takata J, Kataoka Y and Katamine S: Hot spots in prion protein for pathogenic conversion. Proc Natl Acad Sci USA 104: 11921-11926, 2007.
11. Friedler A, Veprintsev DB, Hansson LO and Fersht AR: Kinetic instability of p53 core domain mutants: implications for rescue by small molecules. J Biol Chem 278: 24108-24112, 2003.

12. Foster BA, Coffey HA, Morin MJ and Rastinejad F: Pharmacological rescue of mutant p53 conformation and function. Science 286: 2507-2510, 1999.

13. Mateu MG, Sánchez Del Pino MM and Fersht AR: Mechanism of folding and assembly of a small tetrameric protein domain from tumor suppressor p53. Nat Struct Biol 6: 191-198, 1999.

14. Nikolova PV, Wong KB, DeDecker B, Henckel J and Fersht AR: Mechanism of rescue of common p53 cancer mutations by second-site suppressor mutations. EMBO J 19: 370-378, 2000.

15. Bullock AN and Fersht AR: Rescuing the function of mutant p53. Nat Rev Cancer 1: 68-76, 2001.

16. Bykov VJ, Issaeva N, Shilov A, Hultcrantz M, Pugacheva E, Chumakov P, Bergman J, Wiman KG and Selivanova G: Restoration of the tumor suppressor function to mutant $\mathrm{p} 53$ by a low-molecular-weight compound. Nat Med 8: 282-288, 2002.

17. Rippin TM, Bykov VJ, Freund SM, Selivanova G, Wiman KG and Fersht AR: Characterization of the p53-rescue drug CP-31398 in vitro and in living cells. Oncogene 21: 2119-2129, 2002.

18. Cheng Y, LeGall T, Oldfield CJ, Mueller JP, Van YY, Romero P, Cortese MS, Uversky VN and Dunker AK: Rational drug design via intrinsically disordered protein. Trends Biotechnol 24: 435-442, 2006.

19. Joerger AC, Ang HC and Fersht AR: Structural basis for understanding oncogenic p53 mutations and designing rescue drugs. Proc Natl Acad Sci USA 103: 15056-15061, 2006.

20. Vassilev LT, Vu BT, Graves B, Carvajal D, Podlaski F, Filipovic Z, Kong N, Kammlott U, Lukacs C, Klein C, Fotouhi N and Liu EA: In vivo activation of the p53 pathway by smallmolecule antagonists of MDM2. Science 303: 844-848, 2004.

21. Park SE, Min YK, Ha JD, Kim BT and Lee WG: Novel small molecule induces p53-dependent apoptosis in human colon cancer cells. Biochem Biophys Res Commun 358: 842-847, 2007.

22. Wang W, Kim SH and El-Deiry WS: Small-molecule modulators of p53 family signaling and antitumor effects in p53-deficient human colon tumor xenografts. Proc Natl Acad Sci USA 103: 11003-11008, 2006.

23. Ventura A, Kirsch DG, McLaughlin ME, Tuveson DA, Grimm J, Lintault L, Newman J, Reczek EE, Weissleder R and Jacks T: Restoration of p53 function leads to tumour regression in vivo. Nature 445: 661-665, 2007.

24. Cho Y, Gorina S, Jeffrey PD and Pavletich NP: Crystal structure of a P53 tumor suppressor-DNA complex: understanding tumorigenic mutations. Science 265: 346-355, 1994.

25. Irvin JJ and Shoichet BK: ZINC-a free database of commercially available compounds for virtual screening. J Chem Inf Model 45: $177-182,2005$.

26. Morris GM, Goodsell DS, Halliday RS, Huey R, Hart WE, Belew RK and Olson J: Automated docking using a Lamarckian genetic algorithm and an empirical binding free energy function. J Comput Chem 19: 1639-1662, 1998.

27. Boeckler FM, Joerger AC, Jaggi G, Rutherford TJ, Veprintsev DB and Fersht AR: Targeted rescue of a destabilized mutant of p53 by an in silico screened drug. Proc Natl Acad Sci USA 105: 10360-10365, 2008. 